\title{
STF-2 Cryomodule Performance and New Input Coupler R\&D for ILC
}

\author{
Yasuchika Yamamoto ${ }^{1}$ \\ High Energy Accelerator Research Organization (KEK) \\ 1-1 Oho, Tsukuba-shi, Ibaraki-ken, Japan \\ E-mail: yasuchika.yamamoto@kek.jp
}

T. Honma, E. Kako, Y. Kojima, T. Matsumoto, S. Michizono, H. Nakai, T. Shishido, A. Yamamoto

High Energy Accelerator Research Organization (KEK)

1-1 Oho, Tsukuba-shi, Ibaraki-ken, Japan

\section{Julie, E. Montesinos}

CERN

CH-1211, Geneva 23, Switzerland

The high power test for STF-2 cryomodule with twelve cavities has completed successfully in 2015. It became clear the average accelerating gradient was $30.3 \mathrm{MV} / \mathrm{m}$, eight cavities achieved above $31.5 \mathrm{MV} / \mathrm{m}$ as the ILC specification, and three cavities had the significant performance degradation by heavy field emission due to the additional clean room work in the STF tunnel. As the following next steps, there are the LFD (Lorenz Force Detuning) measurement, LFD compensation by piezo, and long run for check of stable operation at high gradient. In the long run around $32 \mathrm{MV} / \mathrm{m}$, each cavity without degradation showed the stable operation with the successful LFD compensation by piezo and RF feedback system. On the other hand, from the view point of plug-compatibility for the input coupler in the ILC, new STF input couplers with $40 \mathrm{~mm}$ of input port were re-designed, fabricated and high-power-tested successfully. Moreover, for the lower cost study, another input couplers with coating-free ceramic will be also tested soon in the collaboration of CERN and KEK. In this presentation, the detailed results for STF-2 Cryomodule test and $40 \mathrm{~mm}$ input couplers are reported.

38th International Conference on High Energy Physics

3-10 August 2016

Chicago, USA

\footnotetext{
${ }^{1}$ Speaker 


\section{Introduction}

The STF (Superconducting RF Test Facility [1]) was constructed in 2008 to establish the Superconducting RF (SRF) technology for the International Linear Collider (ILC) [2]. The STFtype cavity, coupler, and tuner are KEK original designs. At present, STF Phase-2 (STF-2) is under progress. Figure 1 shows the STF-2 accelerator layout. The goal of STF- 2 is to establish beam operation with the same conditions as those of the ILC (average accelerating gradient of $31.5 \mathrm{MV} / \mathrm{m}$, quality factor $\left(\mathrm{Q}_{0}\right)$ more than $10^{10}$, beam current of $5.8 \mathrm{~mA}$, and so on). The configuration includes an injector cryomodule with two cavities (operating gradient of $20 \mathrm{MV} / \mathrm{m}$ ). The cryomodule is labeled as "Capture CM" in Figure 1, and it was previously installed for the Quantum Beam project [3] in 2012. The STF-2 cryomodules (one and half size cryomodules, called CM1 and CM2a) with 12 cavities were installed downstream of the Capture CM in 2014. The both beamlines upstream and downstream of the STF-2 cryomodules are not constructed yet.

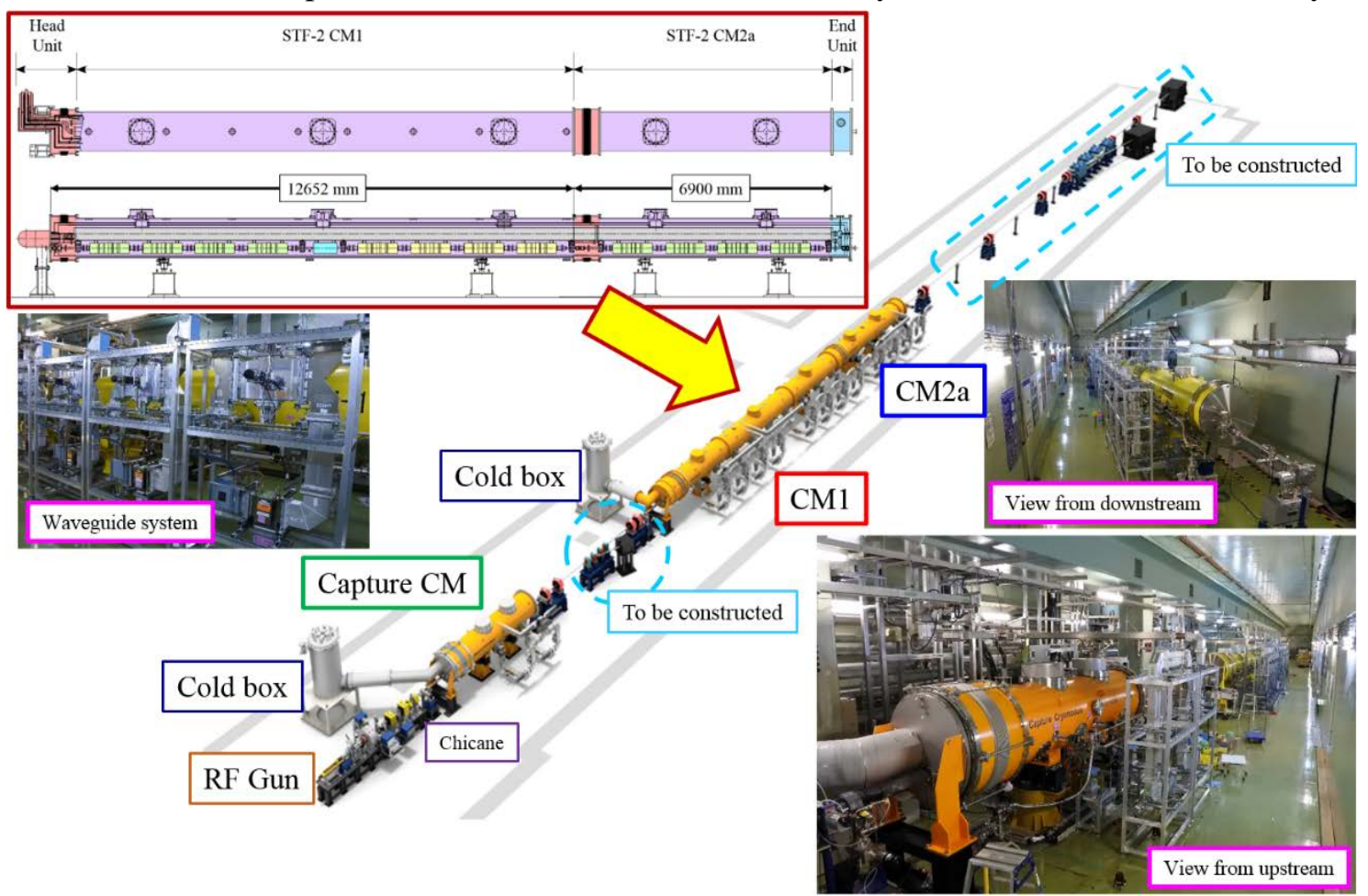

Figure 1: STF-2 Accelerator Layout. "Capture CM" is used as injector cryomdoule in STF-2, installed for Quantum Beam project in 2012. The waveguide system was constructed for eight cavities in the STF-2 cryomodules.

In the Technical Design Report for the ILC, the TTF-3 coupler is described as one candidate. In STF, as original design, the STF-type coupler has been used in cryomodule tests. There are two main different points from the TTF-3 coupler. One is $60 \mathrm{~mm}$ of input port diameter, and the other is narrow range of $\mathrm{Q}_{\mathrm{L}}\left(2-4 \times 10^{6}\right)$. In 2013, the Linear Collider Collaboration recommended the STF-type coupler can be modified for plug-compatible design with $40 \mathrm{~mm}$ of input port diameter. Several STF-type input couplers were newly fabricated for plug-compatible design and additionally lower cost study, assembled in clean room for test bench, and tested with lower power by network analyzer and high power by klystron. 


\section{STF-2 experiment}

Following the first cooldown for low power testing in late 2014 [4], a second cooldown was performed for high power testing in late 2015. The goal of the high power test is to check the cryomodule assembly procedure by comparing the performance of the vertical test (V.T.) and cryomodule test (C.T.).

\subsection{RF conditioning and Cavity performance}

After cooldown of cryomodule to $2 \mathrm{~K}$, the RF conditioning for twelve cavities started from short pulse ( $800 \mu$ s of filling time $/ 100 \mu$ s of flat-top) at first, and after that, moved to full pulse ( $800 \mu$ s of filling time/800 $\mu$ s of flat-top) in the end. The RF conditioning was carefully done monitoring radiation level, heating and RF outputs at HOM (Higher Order Mode) couplers. When RF processing started in cavity, HOM coupler frequently had unusual heating and RF output in each RF pulse. On the other hand, the radiation level, which was measured at three locations near cryomodule, showed rapid increase or decrease repeatedly, and turned to some intensity in the end. Similarly, unusual heating and RF output at HOM couplers gradually disappeared. Figure 2 (left and center) shows one-day-trend-graph of cavity conditioning at $2 \mathrm{~K}$, that is, forwarded power, accelerating gradient, temperatures at both HOM couplers in left figure, unusual output from HOM coupler \#1 in one shot by oscilloscope in center-up, and x-rays measured at three locations in center-down figure. It typically took around 20 hours to do the RF conditioning for each cavity. When the RF processing starts, it is important to keep some gradient level till unusual heating and RF output at HOM couplers disappears completely.
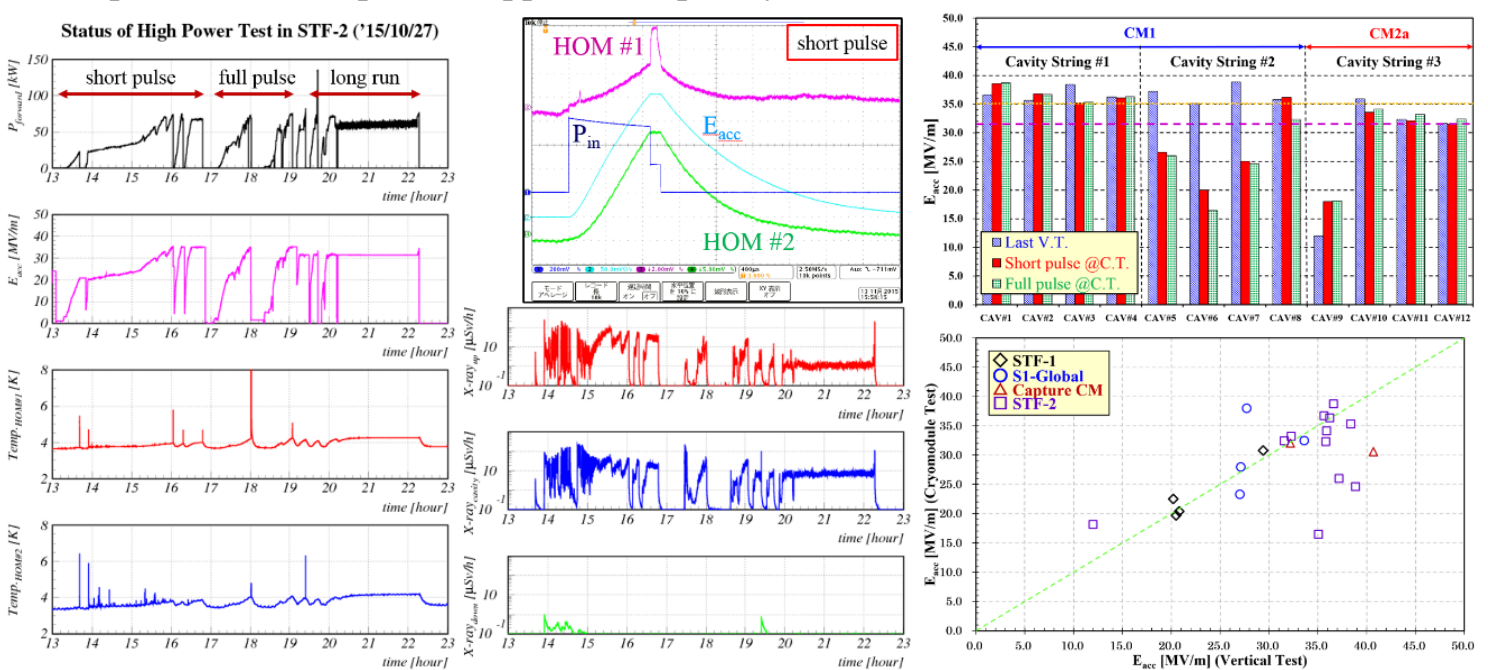

Figure 2: Daily status of RF conditioning for one cavity (left and center), and Cavity performance (right).

After RF processing for each cavity, the maximum accelerating gradient was checked in pulsed mode. Figure 2 (right) shows the result of cavity performance in V.T. and C.T. Cavities \#1-\#4, \#8, and \#10-\#12 had no performance degradation. However, Cavities \#5-\#7 had significant degradation by heavy field emission (\#5 and \#7), and enormous heat loss (\#6). Cavity \#9 already had heavy field emission in V.T.; however, the maximum accelerating gradient in C.T. was improved due to a much lower RF duty factor ( 1\%). This cavity might have had a significant problem during the fabrication process, because a sputtered ball was observed at some distance from the equator region in cell \#9. In addition, Cavities \#9-\#12 were fabricated by a mass 
production scheme (some processes at electron beam welding were drastically changed) at a vendor [5]. Consequently, 8 of 12 cavities achieved above $31.5 \mathrm{MV} / \mathrm{m}$ meeting the ILC specification in C.T.

\subsection{Cause of performance degradation}

Cavities \#5-\#7, which demonstrated significant degradation in C.T., are connected to each other in series, as shown in Figure 2. Therefore, it is thought that the performance degradation was generated by a common cause. Figure 3 shows the local clean booth used for beampipe connection between Cavities \#4 and \#5 in the STF tunnel. Unlike EU-XFEL (European X-ray Free Electron Laser) [6] in DESY, STF-2 cryomodule assembly processes were not straightforward due to insufficient infrastructure capacity at the STF. The main issues are as follows:

- Local clean booth was not of high quality

- Mismatched metal valve between Cavities \#4-\#5

- Argon ventilation when opening gate valves
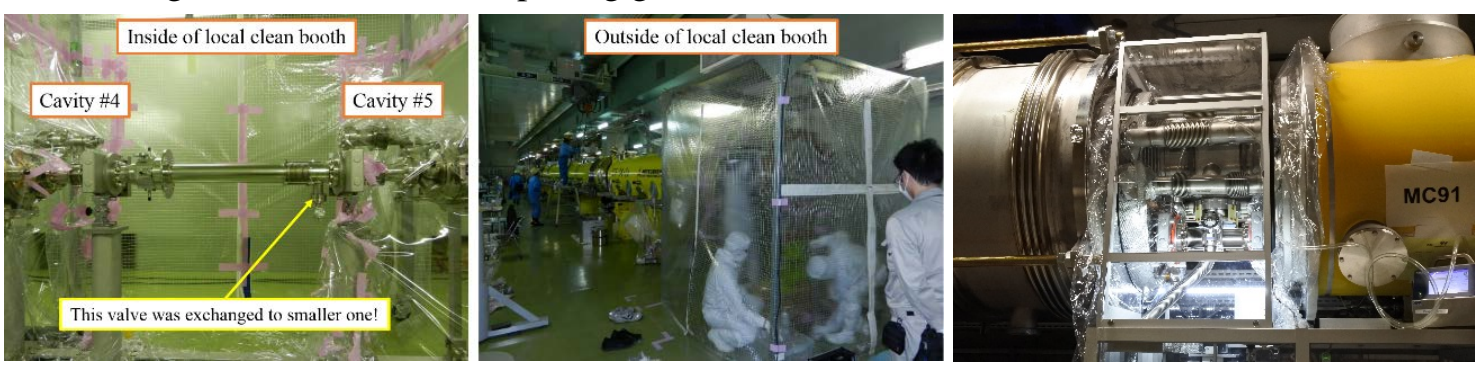

Figure 3: Local clean booth used for beampipe connection in STF tunnel (left and center), and high-quality local clean booth and particle counter used in E-XFEL at DESY (right).

First, as shown in Figure 3, the local clean booth in the STF is large and has a lower flow rate of clean air. This can lead to some contamination during beampipe connection. Second, when the beampipe connection to the side of Cavity \#5 was made, the metal valve needed to be exchanged for a smaller one due to overlapping between the metal valve and thermal shield of the cryomodule. During this work, many dust particles could be generated near Cavity \#5. Third, the gate valve between Cavities \#4 and \#5 was opened under argon ventilation (generally, when opening gate valves, the both beampipe and cavity should be under vacuum). Compared to the case of vacuum, there can be some risk for contamination. Consequently, it is likely that Cavities \#5-\#7 were contaminated by many dust particles, which led to heavy field emission in C.T.

\section{New Input Coupler R\&D}

Following the recommendation by the Linear Collider Collaboration in 2013, new input couplers were re-designed and fabicated to meet the specification in the Technical Design Report. Additionally, two cold parts with ceramic window without Titanium-Nitrogen (TiN) coating were also fabricated for lower cost study. Therefore, there are two warm and cold parts with coated ceramic window, and two cold parts with coating-free ceramic window.

\subsection{Re-design, Fabrication, Inspection and Assembly}

The RF design for input coupler in test stand was done by simulation to meet the wider range of loaded $\mathrm{Q}\left(\mathrm{Q}_{\mathrm{L}}=10^{6-7}\right)$ and input port of $40 \mathrm{~mm}$. Consequently, it was necessary to introduce 
longer tapered pipe and longer bellows for inner conductor. There was no changed point for the other components. Figure 4 shows the schematic view, assembly work in clean room and RF simulation result.

In the incoming inspection after the delivery to KEK, No-Good copper plating for one cold part with TiN coating was observed. At the head of inner conductor, which has the highest electric field during high power test, there were many blisters. However, due to limited schedule, ultrapure water rinsing and assembly work in clean room were done. After assembly work, the coupler test stand had baking at 100 degrees in temperature for 48 hours. The low power test by network analyzer was done to adjust the RF matching condition.

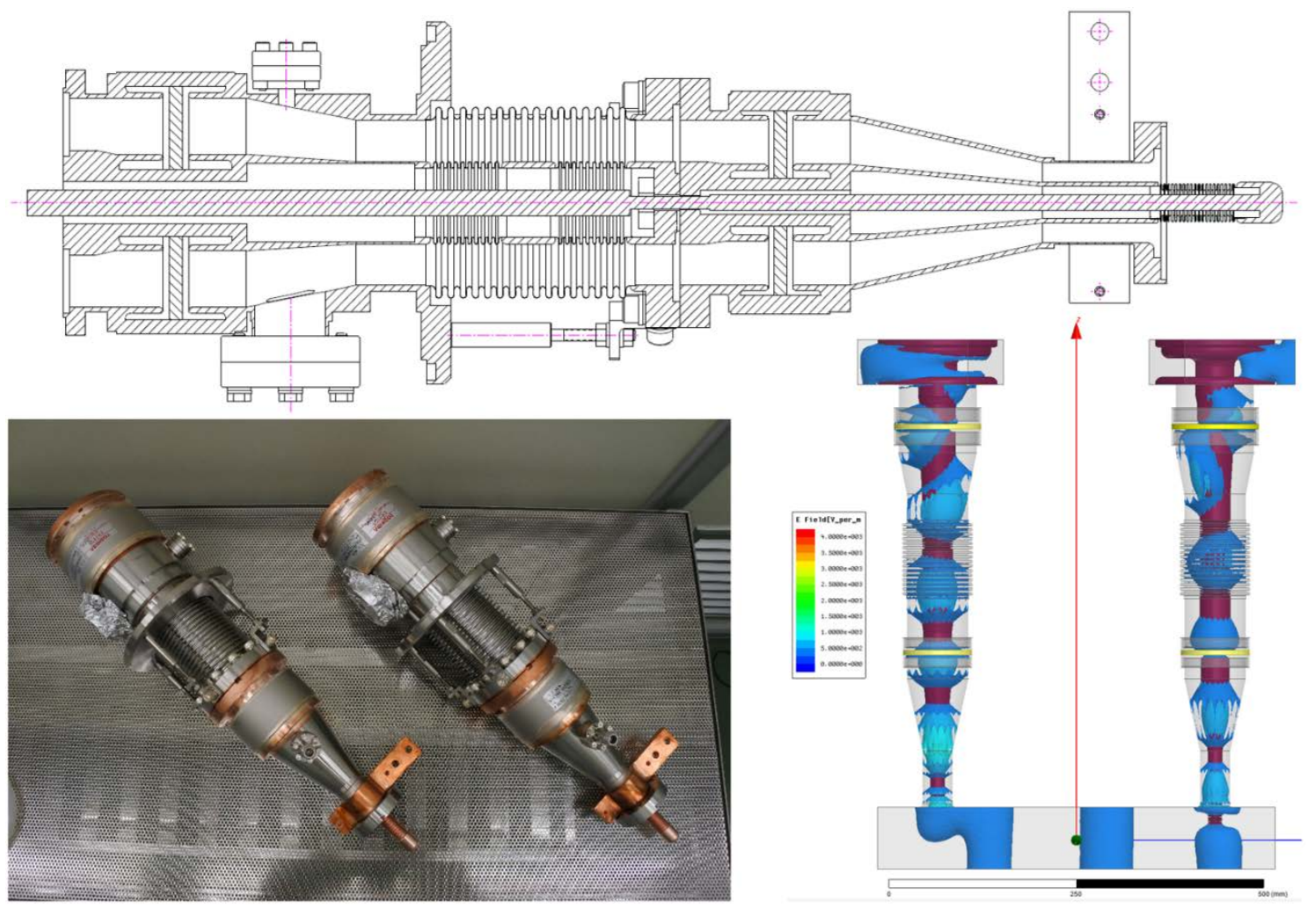

Figure 4: Schematic view of new STF-type input coupler (top), assembly in clean room (bottomleft), and RF simulation by HFSS (bottom-right).

\subsection{Preliminary result}

After baking, the coupler test stand was moved to high power test area in STF, as shown in Figure 5 (left). As for monitoring system, there are warm/cold vacuum, arc discharge sensors, electron emission sensors, forward/backward power from directional couplers at front and end of test stand. Within these items, vacuum, arc, and power signals are used as technical interlock. The vacuum level for technical interlock is $2 \times 10^{-4} \mathrm{~Pa}$. The RF conditioning was done for various pulse widths of 10, 30, 100, 500, 1500 and $1650 \mu$ sec. Generally, the conditioning time for $10 \mu$ sec is dominant. Before the RF conditioning for couplers with coating-free ceramic window, two useful modules were delivered by CERN. One is auto-conditioing module, and the other is vacuum distributor module. The auto-conditioning module can control power level automatically by continually monitoring vacuum level. On the other hand, the vacuum distributor can promptly identify higher vacuum level within warm and cold vacuum. 
The first test was done for couplers with coated ceramic window, and next for couplers with coating-free ceramic window. Figure 5 (right) shows the result for the couplers with coated ceramic window. The total conditioning time was about 92hours, and typical result in STF. As described before, although one of two cold parts had No-Good copper plating at the head of inner conductor, fortunately, there was no unexpected event. Consequently, the plug-compatible design as first goal in this R\&D was successful in high power test. On the other hand, in RF conditioning for couplers with coating-free ceramic window, the conditioning time below $500 \mu \mathrm{sec}$ was shorter, because of the use of same warm parts. However, during RF conditioning in $1500 \mu \mathrm{sec}$, unusual heating was observed at tapered pipe of cold part around $480 \mathrm{~kW}$. At the same time, electron emission suddenly increased up to maximum level $(10 \mathrm{~V})$. As the result of various coupler combination tests, it was clear that only couplers with coating-free ceramic window had unusual heating around the tapered pipe of cold part. Probably, this cause is due to coating-free ceramic window, which has higher secondary electron emission coefficient. At present, the electron tracking simulation by CST Particle Studio is under progress, and will be presented in near future.
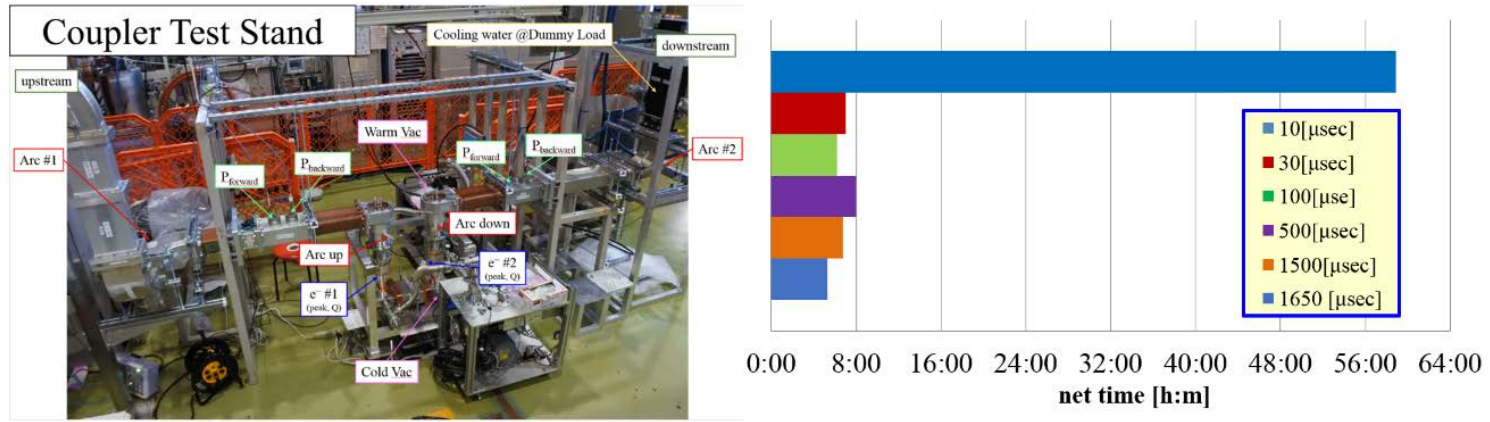

Figure 5: Coupler test stand in STF (left), and RF conditioning time for couplers with coated ceramic window (right).

\section{Summary}

The STF-2 cryomodule test was done to check the performance for twelve cavities. Eight cavities achieved above $31.5 \mathrm{MV} / \mathrm{m}$ as the ILC specification, and however, three cavities had significant degradation. The third cooldown test will be done in this autumn for $\mathrm{Q}_{0}$ measurement and eight cavities operation.

The new R\&D for input coupler started for the plug-compatible design and lower cost study. The plug-compatibility for the STF coupler was successful, and however, coating-free ceramic window made by lower cost had significant problem. It is necessary to develop the other ceramic with new material.

\section{References}

[1] http://www2.kek.jp/stf/

[2] http://www.linearcollider.org/ILC/

[3] Y. Yamamoto et al., in proceedings of IPAC2012, pp. 2236-2238, New Orleans, USA.

[4] T. Shishido et al., in proceedings of SRF2015, TUPB109, Whistler, BC, Canada.

[5] K. Sennyu et al., in proceedings of IPAC2013, pp. 2110-2114, Shanghai, China.

[6] http://xfel.desy.de/ 